\title{
Design of a randomized controlled trial for multiple cancer risk behaviors among Spanish-speaking Mexican-origin smokers
}

Yessenia Castro ${ }^{1}$, Karen Basen-Engquist ${ }^{2}$, Maria E Fernandez ${ }^{3}$, Larkin L Strong ${ }^{4}$, Elizabeth G Eakin ${ }^{5}$, Ken Resnicow ${ }^{6}$, Yisheng $\mathrm{Li}^{7}$ and David W Wetter ${ }^{4 *}$

\begin{abstract}
Background: Smoking, poor diet, and physical inactivity account for as much as 60\% of cancer risk. Latinos experience profound disparities in health behaviors, as well as the cancers associated with them. Currently, there is a dearth of controlled trials addressing these health behaviors among Latinos. Further, to the best of our knowledge, no studies address all three behaviors simultaneously, are culturally sensitive, and are guided by formative work with the target population. Latinos represent 14\% of the U.S. population and are the fastest growing minority group in the country. Efforts to intervene on these important lifestyle factors among Latinos may accelerate the elimination of cancer-related health disparities.

Methods/design: The proposed study will evaluate the efficacy of an evidence-based and theoretically-driven Motivation And Problem Solving (MAPS) intervention, adapted and culturally-tailored for reducing cancer risk related to smoking, poor diet, and physical inactivity among high-risk Mexican-origin smokers who are overweight/ obese $(n=400)$. Participants will be randomly assigned to one of two groups: Health Education (HE) or MAPS (HE + up to 18 MAPS counseling calls over 18 months). Primary outcomes are smoking status, servings of fruits and vegetables, and both self-reported and objectively measured physical activity. Outcome assessments will occur at baseline, 6 months, 12 months, and 18 months.

Discussion: The current study will contribute to a very limited evidence base on multiple risk factor intervention studies on Mexican-origin individuals and has the potential to inform both future research and practice related to reducing cancer risk disparities. An effective program targeting multiple cancer risk behaviors modeled after chronic care programs has the potential to make a large public health impact because of the dearth of evidence-based interventions for Latinos and the extended period of support that is provided in such a program.
\end{abstract}

Trial registration: National Institutes of Health Clinical Trials Registry \# NCT01504919

Keywords: Latinos, Mexican, Mexican American, Smoking cessation, Fruits/vegetables, Physical activity

\section{Background}

Over $60 \%$ of U.S. cancer mortality is attributable to tobacco use, poor nutrition, and physical inactivity [1,2], with almost one-third of all cancers directly attributable to tobacco use alone. Smoking cessation is associated with decreased risk of lung cancer, other cancers, heart attack,

\footnotetext{
* Correspondence: dwetter@mdanderson.org

${ }^{4}$ Department of Health Disparities Research, The University of Texas MD Anderson Cancer Center, Research - Unit 1440, PO Box 301402, Houston, TX 77230-1402, USA

Full list of author information is available at the end of the article
}

stroke, and chronic lung disease [3]. Diet accounts for $35 \%$ of cancer deaths, with diets low in fat, and high in fruits, vegetables, fiber, and grain associated with reduced risk [4-6]. A healthy diet is protective against lung, colon and rectum, breast, oral cavity, esophagus, stomach, pancreas, uterine cervix, and ovary cancer [7]. On the other side of the energy balance equation, regular physical activity can reduce the risk of colon and breast cancer [8-10]. Additionally, balancing "energy in/energy out" (i.e., calories eaten vs. expended) is imperative to avoid weight gain and the increased risk of cancer and other chronic

\section{Biomed Central}


diseases associated with overweight and obesity. Excess body weight has been found to be related to increased risk of as many as 14 types of cancer [11]. Furthermore, these cancer risk factors tend to be clustered, with smokers particularly likely to be physically inactive and have poor diets [12-14]. The presence of multiple cancer risk factors has synergistic adverse effects on health [15]. Thus, strategies for addressing cancer risk reduction among high-risk individuals need to address these multiple health risk behaviors.

The need for effective, culturally tailored behavior change interventions targeted at Latinos is critically important to public health for several reasons. First, Latinos, particularly of Mexican origin, are the fastest growing and largest minority group in the U.S. There are more than 40 million Latinos residing in the US, representing $14 \%$ of the total population [16], and people of Mexican origin account for $59 \%$ of this population [16]. Second, three of the four leading causes of death among Latinos cancer, heart disease, and stroke; [17] share smoking, poor diet, and physical inactivity as risk factors. Third, there are notable disparities in these risk factors for the Latino population. For example, although the prevalence of smoking is lower among Latinos than among the general population $16 \%$ versus $21 \%$; [18], Latinos who smoke are less likely to receive advice to quit from a health professional, and to use cessation counseling services or medication compared to African American or White smokers [19]. National data indicate that $75 \%$ of Latinos do not eat the recommended servings of fruits and vegetables and 58\% are physically inactive, with both Latino men and women being less likely to meet the recommended levels of fruits and vegetables and physical activity compared to non-Hispanic Whites [20]. Fourth, there is a strong clustering of these risk factors among Latinos and disparities exist in this clustering relative to other $\mathrm{racial} / \mathrm{ethnic}$ groups. Latino smokers have both the highest prevalence of overweight/ obesity (79\%) and the highest number of additional risk factors compared to African American or White smokers [21]. Finally, $43 \%$ of Latinos speak Spanish at home and have limited English proficiency, whereas another 36\% speak predominantly Spanish at home [16]. Thus, cancer prevention efforts among Latinos should include Spanish language programs if they hope to have a large public health impact.

Despite the critical importance of reducing cancer risk factors in Latinos, few stringent tests of targeted interventions have been conducted examining behavior change related to smoking cessation, diet or physical activity. For example, in contrast to the hundreds of smoking cessation treatment studies conducted among the general population [22], a recent review found only 12 studies that targeted Latino smokers [23]. Further, although all attended to cultural or linguistic tailoring to some extent, only five of those 12 studies utilized experimental designs. None of these studies attempted to simultaneously affect smoking and another health behavior. The situation is similar for diet and physical activity interventions among Latinos. A recent review found 16 culturally tailored interventions that examined behavioral outcomes (vs. knowledge/attitudes) and were tested with an experimental design [24]. Five of the 16 examined only diet, two examined only physical activity, and eight examined both. One study examined smoking and fruit/vegetable consumption. Five of the 12 smoking interventions and 12 of the 16 diet and physical activity interventions demonstrated significant effects. Thus, although notable efforts to create tailored or targeted interventions for Latinos have been made, there continues to be a dearth of evidence-based interventions for interventions targeting multiple cancer risk behaviors. Interventions addressing multiple cancer risk factors have shown great promise, and there is evidence that attempts to change one risk factor often leads to interest in modifying other risk factors, reflecting potential synergies in changing overall cancer risk profiles [25-28]. In sum, there is a compelling public health need to develop behavior change interventions for Latinos that address multiple cancer risk factors. Further, the development and evaluation of behavior change interventions for Latinos and other underserved groups has been identified as a national health priority $[22,29]$.

\section{Study objective and aims}

The goal of this randomized controlled trial is to evaluate the efficacy of a telephone-based counseling intervention that simultaneously addresses three cancer risk factors (smoking, fruit/vegetable consumption, physical activity) among overweight/obese Mexican-origin smokers in Houston, Texas. The intervention builds on previous work demonstrating the efficacy of the intervention for smoking cessation among Latinos of low socioeconomic status SES; [30], and among female smokers [31], and efficacy in preventing postpartum smoking relapse among a diverse sample of low SES women [32]. Additionally, the intervention is informed by data demonstrating the feasibility of reaching Spanish-speaking Latinos in Texas via a telephone-based intervention [30], and work demonstrating the efficacy of telephone-based interventions for diet and physical activity $[33,34]$.

The specific aims of the study are to: 1 ) test the efficacy of Motivation and Problem Solving (MAPS) to promote and facilitate cancer risk reduction among high-risk Mexican-origin individuals (overweight/obese smokers). Relative to a Health Education (HE) condition, MAPS is hypothesized to result in positive changes in each of the primary smoking, diet, and physical activity outcomes, 
and; 2) assess the effects of MAPS on hypothesized treatment mechanisms (e.g., motivation, agency/self-efficacy, stress/affect) and their potential as treatment mediators.

\section{Motivation and problem solving (MAPS)}

MAPS is a holistic, dynamic framework for behavior change that integrates treatment elements from both motivational interviewing (MI) $[35,36]$ and social cognitive theory $[37,38]$. The overarching theoretical rationale for MAPS is the social cognitive model of behavior change $[38,39]$. Social cognitive theory posits that "High levels of both motivation and self-efficacy are important ingredients ... an individual may fail to engage in a specific behavior despite high levels of self-efficacy if the motivation for performance is low or absent" [38]. That is, theory posits that effective behavior change treatments require both enhancing the motivation to achieve and maintain change, as well as developing the selfefficacy and skills necessary to do so. Similarly, Miller et al. [40] note that "the key element for lasting change is a motivational shift that instigates a decision and commitment to change. In the absence of such a shift, skill training is premature." Nevertheless, current interventions often focus largely on either motivation (e.g., MI-based approaches) or problem-solving/skills training (e.g., social cognitive approaches) despite the strong theoretical and empirical bases for focusing on both. When motivation is addressed, the focus is typically on motivating individuals to initiate behavior change, with little to no focus on the motivation to maintain change or recover from a relapse [22].

MAPS embeds empirically validated social cognitive approaches within an overarching motivational enhancement framework based on MI. MI is a directive but clientcentered therapeutic approach designed to minimize resistance, enhance motivation for change, and increase selfefficacy in a non-confrontational manner [35,36]. Several meta-analyses have supported the efficacy of MI-based interventions for smoking, dietary behavior change, and physical activity [41-44]. MI has been found to be effective for promoting dietary change and physical activity, and our own research has demonstrated the efficacy of a motivational approach (MAPS) for smoking cessation [31,32] including among Spanish speaking Latino smokers [30]. Similarly, the social cognitive model has generated a tremendous amount of intervention research demonstrating that social cognitive treatments for smoking cessation, diet, and physical activity are effective $[45,46]$. However, the relative neglect of motivation reduces their ability to effect behavior change among individuals who are not motivated to change.

Further, although stage-based conceptualizations of behavior change emphasize both motivation and skills training, motivational shifts are conceptualized as relatively stable changes in "stage" [47]. In contrast, MAPS is relatively unique in that it conceptualizes motivation as a fluid construct that can fluctuate on a moment-to-moment basis depending on context. Counselors carefully assess and attend to changes in motivation so that treatment strategies are appropriately matched to motivation in the moment. MAPS utilizes a chronic care model (e.g., extended duration of treatment) and is built around a "wellness program" that in addition to focusing on cancer risk behaviors, also addresses life events, stressors, and other concerns (e.g., depression, family, financial, etc.). By addressing the larger context in which health behaviors occur, not only are many of the barriers for successful behavior change addressed, but adherence is increased because individuals perceive that the counselors care about them as whole people, and are not solely interested in their health behaviors.

\section{Major hypothesized mechanisms}

Both research and theory identify motivation, agency, and stress/negative affect as critical mechanisms underlying behavior change [48-51]. As such, MAPS specifically targets these mechanisms and they are hypothesized to underlie MAPS effects on behavior change.

\section{Motivation}

A large body of evidence supports the role of motivation in the decision to change, the likelihood of change, and the maintenance of change. Motivation, measured in varying ways, predicts smoking quit attempts, smoking cessation success, dietary change, and change in physical activity [47,52-55]. There is also evidence demonstrating that motivation can change rapidly [56-58], consistent with models positing that motivation is dynamic and characterized by frequent fluctuations [58]. Motivation for the maintenance of change has received little attention despite the fact that social cognitive theory posits that "The final and most important stage of the change process is the maintenance stage. It is during the maintenance stage (which begins the moment after the initiation of abstinence or control) that the individual must work the hardest to maintain the commitment to change over time," [38]. More specifically, the motivation for maintaining change may weaken and ambivalence may increase as the individual is exposed to temptations and stressors [38]. Therefore, MAPS includes a specific emphasis on motivation throughout the entire change process and on appropriate therapeutic responses to rapid fluctuations in motivation. In MAPS, the counselor continually attends to motivational cues and adjusts therapeutic strategies in response to even momentary changes in motivation. 


\section{Agency (sense of control, self-efficacy)}

Human agency reflects the ability to intentionally affect one's behavior or life situation. Agency is determined both by personal resources and by the contextual influences impinging on that individual [59]. Concepts encompassed under agency include sense of control and self-efficacy. Sense of control is a learned expectation that outcomes depend on personal choices and actions rather than on chance, other people, or forces outside one's control $[50,60]$. Self-efficacy is a form of agency that is context and behavior dependent; i.e., self-efficacy varies based on the behavior to be performed and situational demands [50,51]. A greater sense of agency is reflected in greater self-efficacy when faced with situations that challenge one's ability to initiate or maintain change. Self-efficacy is a consistent predictor of behavior change in smoking, physical activity, and fruit/vegetable consumption [61-66]. Therefore, based on both data and social cognitive theory [38] MAPS targets agency via the removal of barriers to change, standard problem-solving and coping skills training, and by increasing motivation $[38,67,68]$.

\section{Stress/negative affect}

Stress and negative affect, measured in many different ways, are associated with behavior change [69-71]. In addition, the magnitude and trajectory of stress/negative affect over time are powerful predictors of change $[69,70]$, as are individual differences in affective vulnerability (95-98). Thus, MAPS includes stress management and negative affect reduction strategies.

\section{MAPS adaptation}

MAPS has demonstrated efficacy for smoking cessation among Spanish-speaking Latinos [30], to promote smoking quit attempts among women [31], and preventing postpartum relapse [32]. Thus, the MAPS program must be adapted to additionally address fruit/vegetable consumption and physical activity. Consideration of the needs and preferences of the Mexican-origin population in regards to fruit/vegetable consumption and physical activity must also be addressed during adaptation. Literature review, expert consultation, and focus groups with the target population will guide the adaptation.

\section{Literature review}

A review of the published literature will be conducted with the goal of identifying shared barriers and facilitators of behavior in regards to fruit/vegetable consumption, physical activity, and smoking cessation. The purpose of this is to identify potential targets of intervention common to all three cancer risk behaviors that can be addressed through the MAPS intervention. Literature reviews will also be conducted with the goal of identifying practical models of cultural self-awareness and cross-cultural communication skills to encourage culturally sensitive counselor-participant interactions.

\section{Expert consultation}

Two researchers (authors EGE and KR) who are experts in motivational interventions for diet and physical activity are being consulted for the current study. The purpose of this is to gain expert feedback on the incorporation of these target behaviors into the MAPS intervention, which heretofore has been tested only with regard to smoking [30-32].

\section{Focus groups}

Focus groups will be conducted to gain insights from the members of the target population on exercise and dietary habits, barriers, personal values, and the acceptability of program materials. Five focus groups will be conducted. Focus group participants must meet the same eligibility criteria as the randomized trial. A focus group guide is being developed with input from a subset of the study's community advisory group that included questions about various elements of the interventions and materials. All recommendations for modifications to the interventions and materials will be considered.

\section{Pilot testing}

Following MAPS adaptation, the intervention and study procedures will be pilot tested on up to 20 individuals. Pilot testing will replicate the study procedures through approximately 2-3 months post-baseline assessment. For evaluating the treatments, pilot procedures utilize the "technology model" developed by the Yale Psychotherapy Development Center [72]. Briefly, after developing a good working treatment protocol, the treatment is administered over a shortened timeframe to a small group of participants (typically about $5-7$ ). The counselors and participants are extensively queried during this time as to the appropriateness, acceptability, usefulness, etc. of all materials, assessments, procedures, and counseling. Problems with the protocol usually become apparent fairly quickly and the protocol is then modified based on the feedback and a second round of pilot participants will be enrolled. After 2-3 cycles of pilot testing, the protocols are typically ready to be finalized. At that time, participants will be formally enrolled in the clinical trial. Pilot study participants will be compensated for assessments at a rate commensurate with the formal study participants.

\section{Methods/design \\ Location and setting}

The study site will be the Behavioral Research and Treatment Center of The University of Texas MD Anderson 
Cancer Center. All baseline assessments, outcome assessments, and HE sessions will occur at this location. Additionally, individuals in the MAPS condition will receive telephone counseling based out of the Department of Health Disparities Research at The University of Texas MD Anderson Cancer Center.

\section{Ethics and trial registration}

The current study was funded by National Cancer Institute (NCI) Grant U54CA153505. The study was approved by the Institutional Review Board of The University of Texas MD Anderson Cancer Center (Protocol \#: 20100606), and is registered on the National Institutes of Health Clinical Trials Registry (available at ClinicalTrials. gov. Registration \#: NCT01504919).

\section{Study population and eligibility}

The study population will be overweight or obese Spanish-speaking Mexican- or Mexican-origin smokers who reside in the Houston, Texas, metropolitan area. Inclusion criteria will be: 1) self-report of Mexican heritage; 2) age 18 or older; 3) current smoker with a history of smoking an average of at least one cigarette per day during the last year; 4) register an expired carbon monoxide level of at least five parts per million; 5) body mass index $\geq 25$ based on measured height and weight; 6) ability to engage in low to moderate physical activity as determined by the Physical Activity Readiness Questionnaire PAR-Q; [73]; 7) blood pressure reading <140/90 millimeters of mercury (mm $\mathrm{Hg})$; 8) speak Spanish, and; 9) have a valid home address and a functioning telephone number. Participants do not need to be motivated to change their behavior.

Participants with high blood pressure readings defined as $\geq 140 / 90 \mathrm{~mm} \mathrm{Hg}$; [74] and those deemed ineligible based on the PAR-Q will be able to participate if they provide a letter from a physician who will continue to monitor the participant during the research study. Exclusion criteria include: 1) women who are pregnant or currently lactating; 2) contraindication for nicotine patch; 3) other active substance abuse or dependence; 4) regular use of other tobacco products; 5) current use of tobacco cessation medications; 6) currently enrolled in another study; 6) another household member enrolled in the study, and; 6) a score below 38 on the Short Assessment of Health Literacy for Spanish Adults SAHLSA; [75]. Ineligible individuals will be given referrals to community resources.

\section{Recruitment and consent}

Participants $(\mathrm{N}=400)$ will be recruited from the ongoing Mexican American Cohort Study $(n>20,000)$ in the Houston area and from the community. The Mexican American Cohort study was created in 2001 to assess genetic and non-genetic risk factors for cancer in this population. Recruitment procedures have been described in detail elsewhere [76]. Briefly, participants have been recruited through multiple strategies, including random digit dialing, block walking in predominantly Mexican American neighborhoods (i.e., $>75 \%$ based on 2000 U.S. Census), from community centers and health clinics, and networking through currently enrolled participants. Participants are contacted every 6 months to obtain updated health status and contact information.

For this study, overweight/obese smokers will be identified from the cohort study database as potential participants and will be contacted by research staff. Participants will additionally be recruited via radio and print advertisements and direct community outreach. Potential participants recruited via advertisements will be instructed to call the study telephone line and provide contact information to learn more about the study. All potential participants will be contacted via telephone by research staff to assess their interest in participating. Potential participants will receive a description of the study and will be asked if they are interested in participating. Those who are interested will complete screening over the phone (upon giving verbal consent to be screened), and a baseline visit will be scheduled within two weeks. At the baseline visit, a research coordinator will provide another detailed description of the study, answer questions, and obtain written informed consent to participate in the study. The entire consent process will be completed in Spanish by bilingual research staff, and consent documents will be written in Spanish.

\section{Study design}

The current study is a two-group randomized controlled trial designed to test the efficacy of MAPS versus HE for multiple cancer risk behaviors among Spanish-speaking Mexican-origin smokers. Figure 1 depicts the study flow. Potential participants will call a phone number dedicated to study recruitment. They will undergo an initial eligibility screening (including BMI and smoking status eligibility based on self-report). Those who pass the initial screening and choose to participate will be scheduled to attend an in-person visit at the study site. Additional screening will take place, including objective verification of height, weight, and smoking status. Participants will also be screened for health literacy. After completing additional screening, those who choose to participate will complete the baseline assessment and the first of three HE sessions. After completing the first HE session, participants will be randomly assigned to HE or MAPS.

Randomization will occur using a form of adaptive randomization called minimization $[77,78]$. Compared to techniques such as stratification, minimization results in better group balance on participant characteristics. 


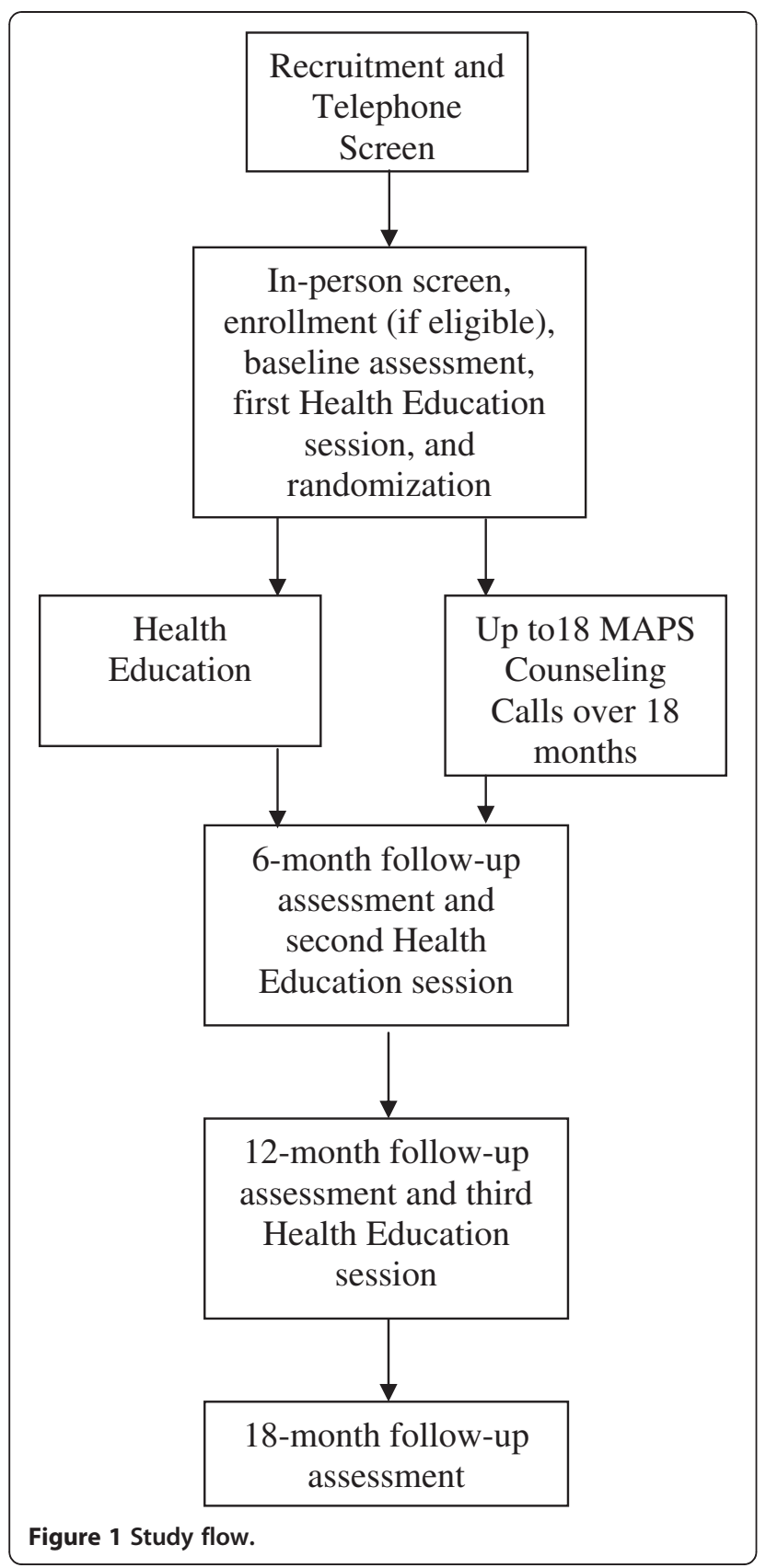

Minimization also provides for balanced treatment groups throughout the randomization process. Randomization will be balanced on gender, age, immigrant status, cigarettes per day, and BMI.

Individuals randomized to the MAPS condition will schedule their first counseling call during the baseline visit, and subsequently receive up to 18 counseling calls over an 18-month period. Outcome assessments will occur at baseline, 6 months, 12 months, and 18 months. Also after completing the assessment and randomization procedures, the 6-month follow-up visit will be scheduled for all participants. Research staff conducting the outcome assessments will be blinded to participants' treatment conditions.

\section{Control and treatment conditions HE condition}

HE will include three sessions of brief advice (10 minutes) regarding smoking cessation, diet, and physical activity change, including the provision of referrals to resources for behavior change (e.g., Texas Quitline, community diet and physical activity programs). Participants will also receive Spanish language self-help materials. Participants will be given a home-based exercise kit (e.g., pedometer, exercise ball, strength training cables) and related instruction on the use of the exercise kit's content during the first $\mathrm{HE}$ session. When the participant informs research staff that $\mathrm{s} /$ he is ready to make a smoking cessation attempt, a 6-week supply of free nicotine patches will be provided. HE will occur a total of 3 times (during the in-person assessment appointments at baseline, 6 months, and 12 months).

\section{MAPS condition}

The treatment condition will include HE plus up to 18 proactive, telephone-delivered MAPS counseling sessions over the 18-month period. Each counseling call will last approximately 20-30 minutes. Call frequency is negotiated between participant and counselor based on a participant's motivation and needs. For example, several calls might be clustered around the initiation of a walking program, a smoking cessation attempt, holiday diet challenges, or specific barriers that the participant is facing (e.g., stress, lack of social support, family problems, financial crisis). Conversely, calls might be scheduled very infrequently when the participant is doing well. Regardless of when calls have been scheduled, participants are always able to call the study telephone line and request a counseling call.

All participants will complete a Wellness Plan in collaboration with their MAPS counselor. The Wellness Plan is an organization of goals or areas of concern/importance that the participant wants to work on during counseling. Wellness Plan items may include change goals for participants who are ready to make changes. The Wellness Plan may also include areas of concern or other topics that the participant would like to discuss where they may or may not be ready to change. Thus, the Wellness Plan will also be used in work with participants who are not motivated to make changes. This is because, although MI will be the primary task being used in working with these participants, it may be helpful to list areas of concern or topics that the participant is simply willing to explore and revisit. Wellness Plan goals can include not only goals related to the target behaviors, but also a plan for addressing other salient 
concerns such as stress, interpersonal issues, family problems, etc. The Wellness Plan can also include plans for connecting participants with resources in the community to address their needs, such as vocational and educational training, and free or low cost childcare and medical treatment. The Wellness Plan is a central organizational component of counseling. Thus, although a participant can develop a Wellness Plan as early as the first few sessions, it is revisited and revised over time to reflect participants' progression through the program or any new areas of emphasis.

MAPS is designed to tailor counseling sessions completely around the participant's expressed readiness to change a target behavior, and their unique needs and preferences for coping skills. Thus, it is unlikely any two participants will progress through the program at the same pace or express/address identical concerns. However, the general progression is broadly organized as follows: Early MAPS counseling sessions (e.g., 1-5) are intended for introduction to the target behaviors and an assessment of the participant's motivation to change any or all of smoking, fruit/vegetable consumption, and physical activity. Where the participant expresses readiness, Problem Solving/Skills Training techniques are utilized to facilitate change. Where the participant is not yet ready, a MI approach is utilized. Later sessions (e.g., 7-16) are used either for continued MI in the areas where the client expresses not being ready to change, action oriented counseling to facilitate change, or maintenance of change, Sessions 17 and 18 prepare the participant for ending the program and include a final review of goals and progress, saying good-bye, and providing any additional necessary referrals.

\section{Counselor qualifications, calls, and training}

MAPS has been developed to be appropriate for delivery by lay and professional health workers. Counselors receive approximately 40 hours of MAPS training initially with "booster" sessions of approximately $1-2$ hours every 1-2 months during the study. Training continues until the counselor reaches competence and adherence criteria. Miscellaneous counseling issues are also discussed at weekly project meetings. This level of training and ongoing monitoring is consistent with the recommended level of training for real-world providers of health promotion interventions such as Quitline counselors [79] and ensures that MAPS is of the highest quality and follows the protocol precisely, both of which are key fidelity issues in evaluating new treatments. Counselors will be two Spanish-speaking members of the study staff who are experienced in the delivery of MAPS.

Treatment fidelity To monitor drift, all calls are digitally recorded and encrypted. A random sample of $10 \%$ of calls are coded using a modified Motivational Interviewing Treatment Integrity Manual MITI; [80]. The MITI has empirically validated reliability and validity, and is modified to include coding around appropriate social cognitive/ problem-solving strategies, and transitions between the motivation building and problem-solving. Because MAPS utilizes a MI framework, the MITI works well for ensuring adherence to the protocol and utilizing the general MI spirit. A counselor who falls below performance criteria will receive additional training.

\section{Instruments}

The majority of the assessment instruments have been previously used and validated in Spanish, or are being tested in our current research. Assessment instruments measure outcomes or hypothesized mechanisms. We attempted to reduce the inconvenience associated with completing the assessment battery by providing financial compensation for participants' time and by using the Questionnaire Design System (QDS), a computer-administered self-interview format. The error and time necessary to complete an assessment battery are substantially reduced by using QDS and particularly so among individuals with low literacy because the computer can read each item in Spanish to the participants. Assessment procedures will be identical at each of the four visits, with the exception that only a subset of the demographic measures will be administered at the 6,12 , and 18 month follow ups.

\section{Primary outcomes \\ Physical activity}

International physical activity questionnaire (IPAQ) The IPAQ assesses walking for exercise, walking for transportation, moderate and vigorous physical activity, and time spent sitting. It is widely used to measure physical activity [73].

Accelerometer Participants will wear a small blinded accelerometer (i.e., participant cannot see values) over 7 days to assess physical activity. Accelerometers will be distributed at each assessment visit and will be returned via mail.

\section{Fruit/vegetable consumption}

National health interview survey diet items Items will be used to assess frequency of food intake in 16 broad categories to estimate intakes of fruits and vegetables, percentage energy from fat, and fiber diets [81].

Two-item food frequency questionnaire The two-item food-frequency questionnaire was developed to estimate intake of fruit and vegetables [82]. 


\section{Smoking abstinence}

Assessments will follow the recommendations from the Society for Research on Nicotine and Tobacco for cessation induction trials [83]. Participants will provide breath carbon monoxide to verify smoking. Although carbon monoxide has a relatively short half-life, the most comprehensive review on biochemical validation concluded that misreporting is typically very low $(\sim 2 \%)$ except for populations with substantial incentives to misreport (i.e., adolescents, pregnant women), and that adjustment for misreporting almost never influences analyses regarding relative treatment efficacy [84]. Participants will also provide a saliva sample to verify smoking status. The saliva sample will be provided at the baseline, 6 month, 12 month and 18 month follow-up visits. Saliva samples will be analyzed for cotinine, a metabolic byproduct of nicotine that provides an estimate of nicotine consumption. A saliva cotinine level of $<20 \mathrm{ng} / \mathrm{ml}$ will be considered as abstinent.

\section{Smoking status questionnaire}

This surveys tobacco use, use of other tobacco products, and nicotine replacement medications. The questionnaire also collects data on the use of other tobacco products and nicotine replacement medications as determined by the participant's time point in the protocol (e.g., based upon the date the participant quits smoking) [83].

\section{Measures of hypothesized treatment mechanisms Barriers self-efficacy physical activity}

The Barriers Self-Efficacy Physical Activity is a 5-item measure on a five-point Likert-type and measures one's confidence to meet a physical activity goal in the face of barriers to the behavior [85].

\section{Fruits and vegetables self-efficacy}

The Fruits and Vegetables Self-efficacy is a 7-item measure on a 5-Likert scales. Items assess family influences, decisional balances, and fruit and vegetable intake [85].

\section{Fruits and vegetables staging}

This is a 2-item measure of daily intake of fruits and vegetables and intent to change the current intake of fruit and vegetables.

\section{Motivation}

Motivation to abstain from smoking will be assessed with a 10-item scale currently being developed by our research team in order to assess motivation as a continuous variable as opposed to a stage. Items assess the motivation to quit smoking [86].

\section{Positive and negative affect scale (PANAS)}

The PANAS is comprised of two mood scales with high reliability, Positive Affect (PA) and Negative Affect (NA) [87].

\section{Self-efficacy scale}

This is a 9-item scale reflecting the confidence of the individual that they can cope with high-risk situations without smoking [88].

\section{Smoking self-efficacy/confidence}

The Smoking Self-Efficacy/Confidence scale assesses an individual's confidence to abstain from substance use or health behaviors in a variety of different situations. It consists of 9-item with 5 responses ranging from not at all confident to completely confident [88].

\section{Smoking status questionnaire}

This surveys tobacco use, use of other tobacco products, and nicotine replacement medications. The questionnaire also collects data on the use of other tobacco products and nicotine replacement medications as determined by the participant's time point in the protocol (e.g., based upon the date the participant quits smoking) [83].

\section{Stages of change: physical activity}

The Stages of Change: Physical Activity is a 1-item measure of the five stages of change in physical activity [89].

\section{Other measures}

\section{Brief Wisconsin Inventory of Dependence Motives}

This questionnaire yields an overall dependence score as well as subscale scores for other critical dimensions of tobacco dependence (affiliative attachment, automaticity, loss of control, cognitive enhancement, craving, cue exposure/ associative processes, social/environmental goads, taste, tolerance, weight control, and affective enhancement). The overall and subscale scores have high internal consistencies and predict abstinence [90].

Demographics Questionnaire collects data on age, race, ethnicity, education, income, preferred language, and generations in the U.S.

\section{Medical history}

Participants will be asked to provide a detail medical history including heart disease, asthma/lung disease, high blood pressure, diabetes, high cholesterol, thyroid problems, and kidney disease.

\section{Medication worksheet}

Participants will be asked to provide a detail list of medications and their dosage that they take daily.

\section{Short assessment of health literacy for Spanish adults (SAHLSA)}

The SAHLSA will be used to assess Spanish language health literacy. Participants are presented with a list of 50 common medical terms. Each word is presented with a synonym and a distracter word. The participant is 
instructed to pronounce each word and identify the synonym. A score of 38 is indicative of at least marginal health literacy, and higher scores indicate greater health literacy [75].

\section{Pharmacotherapy}

All study participants will have access to free nicotine patch therapy when they are ready to make a quit attempt. The provision of patch therapy or other cessation medication is the recommended standard of care. As such, it is arguably an ethical obligation to provide all participants with access to patch therapy. Study participants receive nicotine patch therapy because the patch is currently recognized as a frontline therapy [22], and compared to nonnicotine medications such as bupropion or varenicline, the patch is safer, better tolerated, and available over the counter. Participants who decide to make a quit attempt will request patches by contacting project staff. Patches and instructions for their use will be distributed either via a visit to MD Anderson or via the mail. Patch therapy for participants who smoke $>10$ cigarettes/day will consist of 4 weeks of $21 \mathrm{mg}$ patches, 1 week of $14 \mathrm{mg}$ patches, and 1 week of $7 \mathrm{mg}$ patches. Patch therapy for participants who smoke 6-10 cigarettes/day will consist of 4 weeks of $14 \mathrm{mg}$ patches and 2 weeks of $7 \mathrm{mg}$ patches. Patch therapy for participants who smoke 1-5 cigarettes/day will consist of 6 weeks of $7 \mathrm{mg}$ patches. A reduction in dosage or cessation of the patch regimen will be implemented for any participants who report signs of being on too high of a dose, although this is typically not necessary for participants because blood nicotine levels are usually far lower on the patch than while smoking [91]. In sum, all participants, regardless of treatment group assignment, will have the same access to nicotine patch therapy. We will carefully track pharmacotherapy use at all followup assessments.

\section{Participant compensation and retention}

To compensate for the time and inconvenience associated with participation, participants will be reimbursed $\$ 25$ for each of the 4 assessments (i.e., up to a total of $\$ 100)$. Focus group participants will receive a gift card worth $\$ 50$. Pilot study participants will receive a $\$ 25$ gift card at each visit (Day 1 and Month 3), for a total of up to $\$ 50$ in gift cards. Participants will not receive compensation for the counseling calls. Other procedures to reduce attrition include: 1) mailing postcard reminders and calling to remind participants of upcoming visits; 2) maintaining communication with participants throughout the study via birthday cards, holiday cards, etc. (each mailing includes a stamped address update postcard to update contact information); 3) having research staff member available during daytimes, evenings, and weekends to conduct study visits; 4) requiring that in addition to a functional phone number (necessary for counseling calls), participants have a home address so that they can be contacted by mail if necessary; and 5) obtaining names, addresses, and phone numbers of up to three collaterals (i.e., relatives/friends) who can provide information on participants' whereabouts during the study (permission to contact the collaterals will be obtained from participants).

\section{Data analysis}

Primary outcomes used to evaluate the efficacy of the MAPS intervention (Aim 1) are smoking status, servings of fruits and vegetables, and both self-reported and objectively measured physical activity assessed at the 6,12 , and 18 months. Because the primary outcomes and mechanisms include repeated measurements that are correlated within subjects, the data analytic approach utilizes generalized linear mixed model regression GLMM; [92,93]. Model diagnostics will be used to determine and address the form of the covariances, transformations, collinearity, and influential observations. GLMM parameter tests will be conducted using Wald statistics, and will be adjusted for multiple comparisons where appropriate. Adjustments for multiple comparisons will be made according to the method of Westfall and Young [94]. In this approach, the correlations among the dependent variables are used to adjust the critical values of the individual tests to ensure that the probability of a Type I error across a set of tests does not exceed the chosen alpha level. This approach has the advantage of maintaining the chosen Type I error rates while at the same time providing a less conservative adjustment than Bonferonni-type procedures.

\section{Aim 1}

Evaluate the efficacy of a MAPS approach to promoting and facilitating reduction of behavioral risk factors for cancer among high-risk Mexican-origin individuals (overweight/obese smokers). Relative to HE, MAPS is hypothesized to result in positive changes in each of the primary smoking, diet, and physical activity outcomes.

GLMM will be utilized in analyzing the effects of MAPS on the primary outcomes across the 6, 12, and 18-month time points. For the dichotomous outcome, we will assume a logit link and binomial variance function for the GLMM, and parameterize them with blocking on individual nested within treatment condition. Treatment and time will be included, as well as their interaction, with adjustment for relevant covariates as necessary. Time will be treated as a categorical variable. These models test for the main effects of treatment and time, and whether the treatment effect varies over time. For the continuous outcomes, the GLMM analysis will be replaced by linear mixed model (LMM) analysis 
(i.e., a special case of GLMM in which each outcome variable is continuous).

\section{Aim 2}

Assess the effects of MAPS on hypothesized treatment mechanisms (e.g., motivation, agency/self-efficacy, stress/ affect) and the role of those mechanisms in mediating the effect of MAPS on outcomes.

To assess MAPS effects on treatment mechanisms, analyses largely analogous to the GLMM and LMM analyses described for Aim 1 will be conducted. Mediation will be indicated if: (a) there is a significant MAPS effect on the mechanism, and (b) the mechanism significantly predicts the outcome when adjusting for treatment. To formally test for the mediation effect, we will follow appropriate methods described by MacKinnon [95]. In particular, we will use a product-of-coefficients approach in which the indirect (mediation) effect is defined as the product of the coefficient of the intervention condition in the regression of the mechanism ( $a$ path) and the coefficient of the mechanism in the regression of the outcome variable controlling for intervention condition ( $b$ path), with appropriate coefficient standardization from $b$ path, when the outcome is binary [95]. This approach is applicable for both continuous and binary outcomes (e.g., smoking status, servings of fruits and vegetables, and both selfreported and objectively measured physical activity), with and without repeated measurements. We will use a bootstrapping approach to computing confidence intervals of the indirect effects. Multiple mediator models will be fit to assess simultaneous mediation effects by multiple mechanisms. In addition to the tests for indirect effects, we will also calculate proportion mediated effects and standard errors in both the simple and multiple mediator models.

Missing data and drop-outs Some individuals will fail to complete all assessments. GLMM is designed to handle missing data and will give valid inferences for effects provided that the probability of missing data depends only on the observed outcome and/or covariates in the model (or data are missing at random or missing completely at random). We will conduct analyses to examine whether participants who drop out of the study differ from those who do not, and control for those characteristics that are unbalanced between dropouts and completers and believed to be associated with the outcomes. In case where non-ignorable dropout [96] is suspected, our primary analysis approach will use a conservative one of coding the missing outcome as a failure. For example, a missing smoking outcome could be coded as smoking. In addition, we will conduct sensitivity analysis using selection models to account for nonignorable dropouts. Specifically, we will follow the approach of Diggle and Kenward [97] to model the dropout as a function of both the currently unobserved and previously observed values of the outcome variables (e.g., when dropout is due to lack of improvement in the outcome). Alternatively, we can consider a slopedependent dropout mechanism $[98,99]$ to account for non-ignorable missingness. In this model, participants are believed to drop out with a high probability if the underlying (unobserved) rate of their change of outcome (e.g., physical activity level) over time is low. In spite of the availability of these potentially useful missing-data handling techniques, we do not expect they will lead to remarkably different results or conclusions given our consistently high follow-up rates [30,100-103].

\section{Power}

All power analyses assume a significance level of 0.05 and a two-sided test and accounts for a potential $20 \%$ attrition at all time points $(\mathrm{N}=320 ; 160$ /group). Three of the outcomes are continuous (servings of fruits and vegetables, METS of physical activity, accelerometer assessed activity). One outcome (smoking status) is dichotomous. Power is expected to be greater for intent-to-treat analyses than the power reported here, which represents completers only analyses. This is because the total number of cases for intent-to-treat analyses will be 400 , versus 320 for the completers only analyses. For intent-to-treat analyses, those lost to follow up will be coded as not abstinent.

\section{Continuous outcomes}

Because treatment outcomes are measured at 6,12 , and 18 months, treatment effects may not be consistent across time points. In the case of a constant treatment effect across time, the detectable difference depends on the intraclass correlation coefficient (ICC) between measurements taken from a given participant. Since 160 participants per arm are assumed after attrition, and there are 3 measurements per participant, the effective sample size (ESS) per arm is 3*160/VIF, where VIF is the variance inflation factor $1+(\mathrm{p}-1) * \mathrm{ICC}$. In this formula, $\mathrm{p}$ is the average number of observations per participant. Table 1 shows the minimal detectable constant treatment effect under a range of values for the ICC when testing at a power of $80 \%$. The detectable differences are given in terms of the standard deviation of the error term of the model. Thus, if the ICC is 0.3 , the current study has $80 \%$ power to detect a shift of 0.229 standard deviations between the treatment arms, a small effect size per Cohen [104]. Power calculations displayed in Table 1 assume approximately consistent effects of treatment across measurements. However, correlations between more distal time points are generally lower than 
more proximal time points. Thus, calculations presented in Table 1 represent a worst-case scenario. We further computed power for detecting an average intervention difference of 0.3 when the effect varies across time points, specifically, with a shift of 0.1 at 6 months, 0.3 at 12 months, and 0.5 at 18 months. A Geisser-Greenhouse Corrected $\mathrm{F}$ Test was used for testing the intervention effect. The estimated power for detecting the treatment effect was $99 \%, 96 \%$, and $91 \%$ for ICC values of $0.1,0.3$, and 0.5 , respectively. It is important to note that the presented case of a $0.1,0.3$, and 0.5 increasing difference across time points produces exactly the same power as that of a $0.5,0.3$, and 0.1 decreasing difference across those time points.

\section{Binary outcomes}

National data suggest that approximately $5 \%$ of the general population of smokers quit each year [105]. However, smokers in HE receive a brief treatment based on national recommendations [22]. Therefore, we estimate that the abstinence rate for HE will be approximately $5 \%, 10 \%$, and $15 \%$ across the three follow-ups (i.e., $10 \%$ quit rate per year). Power calculations for correlated binary outcomes are more complex than continuous outcomes, which require simulations. For example, it is not possible to have the same ICC between all pairwise observations. Therefore, the mean of all pairwise ICC values was used as the overall ICC and looked at three potential scenarios based on the simulation of 1000 trials. Using the estimated quit rate for $\mathrm{HE}$ of $5 \%, 10 \%$, and $15 \%$ across the three follow-ups, Table 2 shows simulation results for constant, increasing, and decreasing differences between arms for each time point at ICC values of $0.1,0.3$, and 0.5 . For cessation induction trials such as this one, the most likely scenario would be increasing differences over time given that $80-90 \%$ of smokers will not be motivated to quit at Baseline. Regardless, power is very good for detecting reasonable and meaningful differences between groups under realistic scenarios.

$\begin{aligned} & \text { Table } \mathbf{1} \text { Minimal detectable difference with constant } \\
& \text { effect }\end{aligned}$
\begin{tabular}{|lccc}
\hline ICC & VIF & ESS & Detectable difference \\
\hline 0.0 & 1.0 & 480 & 0.181 \\
0.1 & 1.2 & 400 & 0.198 \\
0.3 & 1.6 & 300 & 0.229 \\
0.5 & 2.0 & 240 & 0.256 \\
1.0 & 3.0 & 160 & 0.314 \\
\hline
\end{tabular}

Note: ICC = intraclass correlation coefficient; $\mathrm{VIF}=$ Variance Inflation Factor; $\mathrm{ESS}=$ Estimated Sample Size.

\section{Discussion}

Interventions addressing multiple cancer risk factors have shown great promise, [25-27], and a variety of approaches and settings have been utilized to address multiple cancer risk factors. For example, both worksite and health center based multiple risk factor interventions have shown efficacy with respect to improving diet and physical activity, but smoking cessation results have generally not been as positive [25,26,106-109]. Overall, multiple risk factor interventions provide an exceptionally promising and efficient means by which to facilitate behavior change and reduce cancer risk. Further, telephone-based interventions have demonstrated efficacy and broad reach as a method of of delivering smoking cessation, physical activity and diet/nutrition interventions [30-34]. The proposed study builds on this work using an intervention approach that has already been proven effective addressing smoking [30]. This intervention will be adapted, through literature review, expert consultation, and qualitative work, to simultaneously address smoking cessation, fruit/vegetable consumption, and physical activity, and to be sensitive to the needs of the target population. To the best of our knowledge, the current study will be among the first multiple risk factor intervention studies to focus on Mexican-origin individuals in general, and more specifically on overweight/ obese Mexican-origin smokers, an extremely high risk group.

MAPS combines two widely utilized and empirically validated approaches $[36,39]$ into a comprehensive, proactive, holistic approach that is specifically tailored to the motivational state, life concerns, and needs/preferences of the target population. MAPS is built around an individualized "wellness program" that is based on both successful chronic care models, patient navigation programs, and feedback from underserved communities. In addition to providing the necessary long-term support and strategies to initiate and maintain change, such an approach could potentially be more cost-effective than shorter interventions because of its effects on increasing the durability of treatment effects. Despite enthusiasm for more chronic care type approaches to behavior change, there have been few attempts to actually develop such programs. Moreover, telephone-based multiple risk factor counseling can both reduce the total number of risk factors, and be most effective among individuals with the greatest risk i.e., among individuals with larger numbers of risk factors; [26]. Further, treating cancer risk factors within a chronic care model, as MAPS does, has the potential to boost long-term success rates by improving the ability of an intervention to promote motivation, aid change attempts, prevent relapse, and encourage recycling among individuals who are unsuccessful [110-113]. 
Table 2 Simulation results

\begin{tabular}{|c|c|c|c|c|c|c|c|c|c|c|c|}
\hline & \multicolumn{3}{|c|}{ Constant differences } & & \multicolumn{3}{|c|}{ Increasing differences } & & \multicolumn{3}{|c|}{ Decreasing differences } \\
\hline & 6 months & 12 months & 18 months & & 6 months & 12 months & 18 months & & 6 months & 12 months & 18 months \\
\hline $\mathrm{HE}$ & 0.05 & 0.10 & 0.15 & $\mathrm{HE}$ & 0.05 & 0.10 & 0.15 & HE & 0.05 & 0.10 & 0.15 \\
\hline MAPS & 0.15 & 0.20 & 0.25 & MAPS & 0.10 & 0.20 & 0.30 & MAPS & 0.20 & 0.20 & 0.20 \\
\hline difference & 0.10 & 0.10 & 0.10 & difference & 0.05 & 0.10 & 0.15 & difference & 0.15 & 0.10 & 0.05 \\
\hline ICC & power & \multicolumn{2}{|c|}{$95 \% \mathrm{Cl}$} & ICC & power & \multicolumn{2}{|c|}{$95 \% \mathrm{Cl}$} & ICC & power & \multicolumn{2}{|c|}{$95 \% \mathrm{Cl}$} \\
\hline 0.10 & 0.99 & \multicolumn{2}{|c|}{$0.98-1.00$} & 0.10 & 0.99 & 0.98 & 1.00 & 0.10 & 0.99 & \multicolumn{2}{|c|}{$0.98-1.00$} \\
\hline 0.30 & 0.94 & \multicolumn{2}{|c|}{ 0.93-0.95 } & 0.30 & 0.95 & 0.94 & 0.96 & 0.30 & 0.98 & \multicolumn{2}{|c|}{ 0.97-0.99 } \\
\hline 0.50 & 0.79 & \multicolumn{2}{|c|}{$0.76-0.82$} & 0.50 & 0.84 & 0.82 & 0.86 & 0.50 & 0.98 & \multicolumn{2}{|c|}{$0.97-0.99$} \\
\hline
\end{tabular}




\section{Competing interests}

The authors declare that they have no competing interests.

\section{Authors' contributions}

DWW conceived of the study and drafted the manuscript; YC lead the intervention adaptation efforts and drafted the manuscript; MEF and LLS assisted with adaptation efforts; EGE and KR consulted on the study design and adaptation efforts; YL conducted the power analyses. All authors consulted on study design and approved the final manuscript.

\section{Acknowledgements}

Preparation of this manuscript was supported by grants U54CA153505, P30CA016672, and K01CA157689 from the National Cancer Institute. Its contents are solely the responsibility of the authors and do not necessarily represent the official views of the National Cancer Institute.

\section{Author details}

'School of Social Work, The University of Texas at Austin, Austin, TX, USA. ${ }^{2}$ Department of Behavioral Science, The University of Texas MD Anderson Cancer Center, Houston, TX, USA. ${ }^{3}$ Division of Health Promotion and Behavioral Science, The University of Texas School of Public Health, Houston, TX, USA. ${ }^{4}$ Department of Health Disparities Research, The University of Texas MD Anderson Cancer Center, Research - Unit 1440, PO Box 301402, Houston, TX 77230-1402, USA. ${ }^{5}$ Cancer Prevention Research Centre, School of Population Health, The University of Queensland, Brisbane, QLD, Australia. ${ }^{6}$ Department of Health Behavior and Health Education, University of Michigan School of Public Health, Ann Arbor, MI, USA. ${ }^{7}$ Department of Biostatistics, The University of Texas MD Anderson Cancer Center, Houston, TX, USA.

\section{Received: 14 February 2013 Accepted: 4 March 2013} Published: 18 March 2013

\section{References}

1. Mokdad AH, Marks JS, Stroup DF, Gerberding JL: Actual causes of death in the United States, 2000. JAMA 2004, 291(10):1238-1245.

2. Ries L, Eisner M, Kosary C, Hankey B, Miller B, Clegg L, Mariotto A, Feuer E, Edwards B: SEER Cancer Statistics Review, 1975-2001. Bethesda, MD: National Cancer Institute; 2004

3. United States Department of Health and Human Services: The Health Benefits of Smoking Cessation: A Report of the Surgeon General. Rockville, MD: United States Department of Health and Human Services, Public Health Service, Centers for Disease Control and Prevention, Center for Chronic Disease Prevention and Health Promotion, Office on Smoking and Health; 1990.

4. Danaei G, Vender Hoorn S, Lopez AD, Murray CJL, Ezzati M: Causes of cancer in the world: comparative risk assessment of nine behavioural and environmental risk factors. Lancet 2005, 366(9499):1784-1793.

5. Doll R, Peto R: The causes of cancer: quantitative estimates of avoidable risks of cancer in the United States today. J Nat Cancer Insitute 1981, 66(6):1191-1308

6. Donaldson MS: Nutrition and cancer: a review of the evidence for an anti-cancer diet. Nutr J 2004, 3:19.

7. Block G, Patterson B, Subar A: Fruit, vegetables, and cancer prevention: a review of the epidemiological evidence. Nutr Cancer 1992, 18(1):1-29.

8. Colditz GA, Cannuscio CC, Frazier AL: Physical activity and reduced risk of colon cancer: implications for prevention. Cancer Cause Control 1997 8(4):649-667.

9. Friedenreich CM, Thune I, Brinton LA, Albanes D: Epidemiologic issues related to the association between physical activity and breast cancer. Cancer 1998, 83(3 Suppl):600-610.

10. Shephard RJ, Futcher R: Physical activity and cancer: how may protection be maximized? Crit Rev Oconogen 1997, 8(2-3):219-272.

11. Basen-Engquist $K$, Chang M: Obesity and cancer risk: recent review and evidence. Cur Oncol Rep 2011, 13(1):71-76.

12. Emmons KM, Marcus BH, Linnan L, Rossi JS, Abrams DB: Mechanisms in multiple risk factor interventions: smoking, physical activity, and dietary fat intake among manufacturing workers. Working well research group. Prev Med 1994, 23(4):481-489.

13. Services USDoHaH: Healthy People 2010. Washington, D.C: Department of Health and Human Services; 2001
14. Chiolero A, Wietlisbach V, Ruffieux C, Paccaud F, Cornuz J: Clustering of risk behaviors with cigarette consumption: a population-based survey. Prev Med 2006, 42(5):348-353

15. Marrero JA, Fontana RJ, Fu S, Conjeevaram HS, Su GL, Lok AS: Alcohol, tobacco and obesity are synergistic risk factors for hepatocellular carcinoma. J Hepatol 2005, 42(2):218-224.

16. Ramirez R: We the People: Hispanics in the United States. Washington, D.C: United States Census Bureau; 2004.

17. Group USCSW: United States Cancer Statistics: 2002 Incidence and Mortality. Atlanta: United States Department of Health and Human Services, Centers for Disease Control and Prevention, and National Cancer Institute; 2005.

18. Centers for Disease Control and Prevention: Cigarette smoking among adults and trends in smoking cessation - United States, 2008. MMWR 2009, 58(44):1227-1232.

19. Centers for Disease Control and Prevention: Quitting smoking among adults-United States, 2001-2010. MMWR 2011, 60(44):1513-1519.

20. Centers for Disease Control and Prevention: Prevalence of fruit and vegetable consumption and physical activity by race/ethnicity-United States, 2005. MMWR 2007, 56(13):301-304.

21. Kendzor DE, Costello TJ, Li Y, Vidrine Jl, Mazas CA, Reitzel LR, Cinciripini PM Cofta-Woerpel LM, Businelle MS, Wetter DW: Race/ethnicity and multiple cancer risk factors among individuals seeking smoking cessation treatment. Cancer Epidemiol Biomarkers Prev 2008, 17(11):2937-2945.

22. Fiore M, Bailey W, Cohen S, Dorfman S, Goldstein M, Gritz E, et al: Treating tobacco use and dependence: Clinical practice guidelines. Rockville, MD: United States Department of Health and Human Services, Public Health Service; 2000.

23. Webb MS, Rodriguez-Esquivel D, Baker EA: Smoking cessation interventions among Hispanics in the United States: A systematic review and mini meta-analysis. Am J Health Promot 2010, 25(2):109-118.

24. Mier N, Ory MG, Medina AA: Anatomy of culturally sensitive interventions promoting nutrition and exercise in hispanics: a critical examination of existing literature. Health Promot Prac 2010, 11(4):541-554.

25. Emmons KM, Linnan LA, Shadel WG, Marcus B, Abrams DB: The working healthy project: a worksite health-promotion trial targeting physical activity, diet, and smoking. J Occup Environ Med 1999, 41(7):545-555.

26. Emmons KM, McBride CM, Puleo E, Pollak Kl, Clipp E, Kuntz K, Marcus BH, Napolitano M, Onken J, Farraye F, Fletcher R: Project PREVENT: a randomized trial to reduce multiple behavioral risk factors for colon cancer. Cancer Epidem Biomarkers Prev 2005, 14(6):1453-1459.

27. Prochaska JO, Velicer WF, Redding C, Rossi JS, Goldstein M, DePue J, Greene GW, Rossi SR, Sun X, Fava JL, Laforge R, Rakowski W, Plummer BA: Stagebased expert systems to guide a population of primary care patients to quit smoking, eat healthier, prevent skin cancer, and receive regular mammograms. Prev Med 2005, 41(2):406-416.

28. Mata J, Silva MN, Vieira PN, Carraça EV, Andrade AM, Coutinho SR, Sardinha $L B$, Teixeira PJ: Motivational "spill-over" during weight control: increased self-determination and exercise intrinsic motivation predict eating selfregulation. Sport Exerc Perform Psychol 2011, 1(S):49-59.

29. Services USDoHaH: Tobacco use among U.S. racial/ethnic minority groupsAfrican Americans, American Indians and Alaskan natives, Asian Americans and Pacific Islanders and Hispanics: A report of the Surgeon General. Atlanta, GA: United States Department of Health and Human Services, Centers for Disease Control and Prevention, National Center for Chronic Disease Prevention and Health Promotion, Office on Smoking and Health; 1998.

30. Wetter DW, Mazas C, Daza P, Nguyen L, Fouladi RT, Li Y, Cofta-Woerpel L: Reaching and treating spanish-speaking smokers through the national cancer Institute's cancer information service. A randomized controlled trial. Cancer 2007, 109(2 Suppl):406-413.

31. McClure JB, Westbrook E, Curry SJ, Wetter DW: Proactive, motivationally enhanced smoking cessation counseling among women with elevated cervical cancer risk. Nicotine Tob Res 2005, 7(6):881-889.

32. Reitzel LR, Vidrine JI, Businelle MS, Kendzor DE, Costello TJ, Li Y, Daza P, Mullen PD, Velasquez MM, Cinciripini PM, Cofta-Woerpel L, Wetter DW: Preventing postpartum smoking relapse among diverse low-income women: a randomized clinical trial. Nicotine Tob Res 2010, 12(4):326-335.

33. Eakin EG, Lawler SP, Vandelanotte C, Owen N: Telephone interventions for physical activity and dietary behavior change: a systematic review. Am J Prev Med 2007, 32(5):419-434. 
34. Goode AD, Reeves MM, Eakin EG: Telephone-delivered interventions for physical activity and dietary behavior change: an updated systematic review. Am J Prev Med 2012, 42(1):81-88.

35. Miller W, Rollnick S: Motivational Interviewing: Preparing People to Change Addictive Behaviors. New York: The Guilford Press; 1991.

36. Miller W, Rollnick S: Motivational Interviewing. 2nd edition. New York: The Guilford Press; 2002.

37. Witkiewitz K, Marlatt GA: Relapse prevention for alcohol and drug problems: that was Zen, this is Tao. Am Psychol 2004, 59(4):224-235.

38. Marlatt GA, Donovan D: Relapse prevention: Maintenance strategies in the treatment of addictive behaviors. 2nd edition. New York: The Guilford Press; 2005.

39. Bandura A: Health promotion by social cognitive means. Health Educ Behav 2004, 31(2):143-164.

40. Miller W, Zweben A, Diclemente C, Rychtarik R: Motivational Enhancement Therapy Manual: A Clinical Research Guide forTherapists Training Individuals with Alcohol Abuse and Dependence. Rockville, MD: United States Department of Health and Human Services, Public Health Service, National Institutes of Health, National Institute on Alcohol Abuse and Alcoholism; 1995.

41. Burke BL, Arkowitz H, Menchola M: The efficacy of motivational interviewing: a meta-analysis of controlled clinical trials. J Consult Clin Psychol 2003, 71(5):843-861.

42. Heckman CJ, Egleston BL, Hofmann MT: Efficacy of motivational interviewing for smoking cessation: a systematic review and metaanalysis. Tob Control 2010, 19(5):410-416.

43. Rubak S, Sandbaek A, Lauritzen T, Christensen B: Motivational interviewing: a systematic review and meta-analysis. Brit J Gen Prac 2005, 55(513):305-312.

44. Martins RK, McNeil DW: Review of motivational interviewing in promoting health behaviors. Clin Psychol Rev 2009, 29(4):283-293.

45. Carroll KM: Relapse prevention as a psychosocial treatment: a review of controlled clinical trials. Exp Clin Psychopharm 1996, 4(1):46-54.

46. Greaves C, Sheppard K, Abraham C, Hardeman W, Roden M, Evans P, Schwarz P, Group TIS: Systematic review of reviews of intervention components associated with increased effectiveness in dietary and physical activity interventions. BMC Pub Health 2011, 11(1):119.

47. Prochaska JO, DiClemente CC, Norcross JC: In search of how people change: applications to addictive behaviors. Am Psychol 1992, 47(9):1102-1114

48. Adler NE, Ostrove JM: Socioeconomic status and health: what we know and what we don't. Annals NY Acad Sci 1999, 896(1):3-15.

49. Gallo LC, Matthews KA: Understanding the association between socioeconomic status and physical health: do negative emotions play a role? Psychol Bull 2003, 129(1):10-51.

50. Mirowsky J, Ross C: Education, personal control, lifestyle and health. Res Aging 1998, 20:415-449.

51. Ross C, Wu C: The links between education and health. Am Sociol Rev 1995, 60:719-745.

52. Sciamanna CN, Hoch JS, Duke GC, Fogle MN, Ford DE: Comparison of five measures of motivation to quit smoking among a sample of hospitalized smokers. J Gen Intern Med 2000, 15(1):16-23.

53. Prochaska JO, DiClemente CC: Stages of change in the modification of problem behaviors. In Progress in Behavior Modification. Edited by Herson M, Eisler R, Miller P. Sycamore, IL: Sycamore Publishing Company; 1992

54. Silva MN, Markland D, Vieira PN, Coutinho SR, Carraça EV, Palmeira AL, Minderico CS, Matos MG, Sardinha LB, Teixeira PJ: Helping overweight women become more active: need support and motivational regulations for different forms of physical activity. Psychol Sport Exerc 2010, 11(6):591-601

55. Fortier MS, Wiseman E, Sweet SN, O'Sullivan TL, Blanchard CM, Sigal RJ, Hogg W: A moderated mediation of motivation on physical activity in the context of the physical activity counseling randomized control trial. Psychol Sport Exercise 2011, 12(2):71-78.

56. Hughes JR, Keely JP, Fagerstrom KO, Callas PW: Intentions to quit smoking change over short periods of time. Addict Behav 2005, 30(4):653-662.

57. Larabie LC: To what extent do smokers plan quit attempts? Tob Control 2005, 14(6):425-428

58. West R, Sohal T: "Catastrophic" pathways to smoking cessation: findings from national survey. BMJ 2006, 332(7539):458-460.

59. Bandura A: Toward a psychology of human agency. Perspect Psychol Sci 2006, 1(2):164-180
60. Mirowsky J, Ross C: Estimating defense and agreement bias from measures of the sense of control: A 2x2 index. Soc Psychol Q 1991, 54:127-141

61. DiClemente CC, Prochaska JO, Fairhurst SK, Velicer WF, Velasquez MM, Rossi JS: The process of smoking cessation: an analysis of precontemplation, contemplation, and preparation stages of change. J Consult Clin Psychol 1991, 59(2):295-304.

62. Cinciripini PM, Wetter DW, Fouladi RT, Blalock JA, Carter BL, Cinciripini LG, Baile WF: The effects of depressed mood on smoking cessation: mediation by postcessation self-efficacy. J Consult Clin Psychol 2003, 71(2):292-301.

63. Shiffman S, Balabanis MH, Paty JA, Engberg J, Gwaltney CJ, Liu KS, Gnys M, Hickcox M, Paton SM: Dynamic effects of self-efficacy on smoking lapse and relapse. Health Psychol 2000, 19(4):315-323.

64. Koring M, Richert J, Parschau L, Ernsting A, Lippke S, Schwarzer R: A combined planning and self-efficacy intervention to promote physical activity: A multiple mediation analysis. Psychol Health Med 2012, 17(4):488-498.

65. Richert J, Reuter T, Wiedemann AU, Lippke S, Ziegelmann J, Schwarzer R: Differential effects of planning and self-efficacy on fruit and vegetable consumption. Appetite 2010, 54(3):611-614.

66. Kreausukon P, Gellert P, Lippke S, Schwarzer R: Planning and self-efficacy can increase fruit and vegetable consumption: a randomized controlled trial. J Behav Med 2012, 35(4):443-451.

67. Davis J, Glaros A: Relapse prevention and smoking cessation. Addict Behav 1986, 11:15-114.

68. Hall SM, Rugg D, Tunstall C, Jones RT: Preventing relapse to cigarette smoking by behavioral skill training. J Consult Clin Psychol 1984, 52(3):372-382

69. Carels RA, Douglass OM, Cacciapaglia HM, O'Brien WH: An ecological momentary assessment of relapse crises in dieting. J Consult Clin Psychol 2004, 72(2):341-348.

70. Burgess ES, Brown RA, Kahler CW, Niaura R, Abrams DB, Goldstein MG, Miller IW: Patterns of change in depressive symptoms during smoking cessation: who's at risk for relapse? J Consult Clin Psychol 2002, 70(2):356-361.

71. Glassman AH, Helzer JE, Covey LS, Cottler LB, Stetner F, Tipp JE, Johnson J: Smoking, smoking cessation, and major depression. JAMA 1990, 264(12):1546-1549.

72. Carroll KM, Nuro K: The Technology Model: An introduction to psychotherapy research in substance abuse. New Haven, CT: Yale University Psychotherapy Development Center, Substance Abuse Center; 1996.

73. Thomas S, Reading J, Shephard RJ: Revision of the physical activity readiness questionnaire (PAR-Q). Can J Sport Sci 1992, 17(4):338-345.

74. National Heart $L$, and Blood Institute: 7th Report of the Joint National Committee on Prevention, Detection, Evaluation, and Treatment of High Blood Pressure. Bethesda, MD: United States Department of Health and Human Services, National Institutes of Health, National Heart, Lung, and Blood Institute; 2003.

75. Lee SY, Bender DE, Ruiz RE, Cho Yl: Development of an easy-to-use spanish health literacy test. Health Serv Res 2006, 41(4 Pt 1):1392-1412.

76. Wilkinson AV, Spitz MR, Strom SS, Prokhorov AV, Barcenas CH, Cao Y, Saunders KC, Bondy ML: Effects of nativity, age at migration, and acculturation on smoking among adult Houston residents of Mexican descent. Am J Pub Health 2005, 95(6):1043-1049.

77. Pocock S: Sequential treatment assignment with balancing for prognostic factors in the controlled clinical trial. Biometrics 1975, 31(1):103-115.

78. Pocock S: Clinician Trials: A Practical Approach. New York: John Wiley and Sons; 1993.

79. Centers for Disease Control and Prevention: Telephone Quitlines: A Resource for Development, Implementation and Evaluation. Atlanta, GA: United States Department of Health and Human Services, Centers for Disease Control and Prevention, National Center for Chronic Disease Prevention and Health Promotion, Office of Smoking and Health; 2004.

80. Moyers T, Martin T, Manuel J, Miller W: The Motivational Interviewing Treatment Integrity (MITI) Code, Version 2.0. Albuquerque, NM: University of New Mexico, Center on Alcoholism, Substance Abuse and the Addictions.

81. Thompson FE, Midthune D, Subar AF, Kahle LL, Schatzkin A, Kipnis V: Performance of a short tool to assess dietary intakes of fruits and vegetables, percentage energy from fat and fibre. Pub Health Nutri 2004, 7(8):1097-1105. 
82. Wardle J, Parmenter K, Waller J: Nutrition knowledge and food intake. Appetite 2000, 34(3):269-275.

83. Hughes JR, Keely JP, Niaura RS, Ossip-Klein DJ, Richmond RL, Swan GE: Measures of abstinence in clinical trials: issues and recommendations. Nicotine Tob Res 2003, 5(1):13-25.

84. Velicer WF, Prochaska JO, Rossi JS, Snow MG: Assessing outcome in smoking cessation studies. Psychol Bull 1992, 111(1):23-41.

85. Hagler AS, Norman GJ, Radick LR, Calfas KJ, Sallis JF: Comparability and reliability of paper- and computer-based measures of psychosocial constructs for adolescent fruit and vegetable and dietary fat intake. J Am Diet Assoc 2005, 105(11):1758-1764.

86. Heppner WL, Ji L, Reitzel LR, Castro Y, Correa-Fernandez V, Vidrine Jl, Li Y, Dolan-Mullen P, Velasquez MM, Cinciripini PM, Cofta-Woerpel L, Greisinger A, Wetter D: The role of prepartum motivation in the maintenance of postpartum smoking abstinence. Health Psychol 2011, 30(6):736-745.

87. Watson D, Clark LA, Tellegen A: Development and validation of brief measures of positive and negative affect: the PANAS scales. $J$ Pers Soc Psychol 1988, 54(6):1063-1070.

88. Velicer WF, Diclemente CC, Rossi JS, Prochaska JO: Relapse situations and self-efficacy: an integrative model. Relapse situations and self-efficacy: an integrative model 1990, 15(3):271-283.

89. Marcus BH, Selby VC, Niaura RS, Rossi JS, Lavallee D, Williams JM, Jones MV: Self-efficacy and the stages of exercise behavior change. In Key Studies in Sport and Exercise Psychology. Edited by Lavallee D, Williams JM, Jones MV. Maidenhead, BRK England: Open University Press; 2008:93-109.

90. Smith SS, Piper ME, Bolt DM, Fiore MC, Wetter DW, Cinciripini PM, Baker TB: Development of the brief wisconsin inventory of smoking dependence motives. Nicotine Tob Res 2010, 12(5):489-499.

91. Palmer KJ, Buckley MM, Faulds D: Transdermal nicotine. A review of its pharmacodynamic and pharmacokinetic properties, and therapeutic efficacy as an aid to smoking cessation. Drugs 1992, 44(3):498-529.

92. MCCulloch C, Searle S: Generalized, Linear, and Mixed Models. New York: John Wiley \& Sons, Inc; 2001

93. Verbeke G, Molenberghs G: Linear Mixed Models for Longitudinal Data. New York: John Wiley \& Sons, Inc; 2000.

94. Westfall P, Young S: Resampling-based Multiple Testing: Examples and Methods for P-value Adjustment. New York: John Wiley \& Sons, Inc; 1993.

95. Mackinnon DP, Lockwood CM, Brown CH, Wang W, Hoffman JM: The intermediate endpoint effect in logistic and probit regression. Clin Trials 2007, 4(5):499-513

96. Little R, Rubin D: Statistical Analysis with Missing Data. 2nd edition. New York: John Wiley \& Sons, Inc; 2002.

97. Diggle R, Kenward M: Informative drop-out in longitudinal data analysis. Appl Stat 1994, 43(1):49-93.

98. Wu M, Bailey K: Estimation and comparison of changes in the presence of informative right censoring: conditional linear model. Biometrics 1989, 45:939-955.

99. Eldridge S, Kerry S: A Practical Guide to Cluster Randomised Trials in Health Services Research. Chichester, West Sussex, UK: John Wiley \& Sons, Ltd; 2012.

100. Wetter DW, Carmack CL, Anderson CB, Moore CA, De Moor CA, Cinciripini PM, Hirshkowitz M: Tobacco withdrawal signs and symptoms among women with and without a history of depression. Exp Clin Psychopharm 2000, 8(1):88-96.

101. Wetter DW, Fiore MC, Baker TB, Young TB: Tobacco withdrawal and nicotine replacement influence objective measures of sleep. $J$ Consult Clin Psychol 1995, 63(4):658-667.

102. Wetter DW, Fiore MC, Young TB, McClure JB, de Moor CA, Baker TB: Gender differences in response to nicotine replacement therapy: objective and subjective indexes of tobacco withdrawal. Exp Clin Psychopharm 1999, 7(2):135-144.

103. Wetter DW, Kenford SL, Smith SS, Fiore MC, Jorenby DE, Baker TB: Gender differences in smoking cessation. J Consult Clin Psychol 1999, 67(4):555-562.

104. Cohen S: Statistical Power Analysis for the Behavioral Sciences. Hhillsdale, NJ: Lawrence Earlbaum Associates, Inc; 1988.

105. Prevention CfDCa: Cigarette smoking among adults-United States, 2000. MMWR 2002, 51(29):642-645.

106. Hunt MK, Stoddard AM, Barbeau E, Goldman R, Wallace L, Gutheil C, Sorensen G: Cancer prevention for working class, multiethnic populations through small businesses: the healthy directions study. Cancer Cause Control 2003, 14(8):749-760.
107. Glasgow RE, Terborg JR, Hollis JF, Severson HH, Boles SM: Take heart: results from the initial phase of a work-site wellness program. Am J Pub Health 1995, 85(2):209-216.

108. Glasgow RE, Terborg JR, Strycker LA, Boles SM, Hollis JF: Take heart II: replication of a worksite health promotion trial. J Behav Med 1997, 20(2):143-161.

109. Emmons KM, Stoddard AM, Gutheil C, Suarez EG, Lobb R, Fletcher R: Cancer prevention for working class, multi-ethnic populations through health centers: the healthy directions study. Cancer Causes Control 2003, 14(8):727-737.

110. Glasgow RE, Orleans $C T$, Wagner EH: Does the chronic care model serve also as a template for improving prevention? Milbank Q 2001, 79(4):579-612. iv-v.

111. Steinberg MB, Schmelzer AC, Richardson DL, Foulds J: The case for treating tobacco dependence as a chronic disease. Ann Intern Med 2008, 148(7):554-556.

112. Bodenheimer T, Wagner EH, Grumbach K: Improving primary care for patients with chronic illness: the chronic care model, Part 2. JAMA 2002, 288(15):1909-1914.

113. Bodenheimer T, Wagner EH, Grumbach K: Improving primary care for patients with chronic illness. JAMA 2002, 288(14):1775-1779.

\section{doi:10.1186/1471-2458-13-237}

Cite this article as: Castro et al.: Design of a randomized controlled trial for multiple cancer risk behaviors among Spanish-speaking Mexicanorigin smokers. BMC Public Health 2013 13:237.

\section{Submit your next manuscript to BioMed Central and take full advantage of:}

- Convenient online submission

- Thorough peer review

- No space constraints or color figure charges

- Immediate publication on acceptance

- Inclusion in PubMed, CAS, Scopus and Google Scholar

- Research which is freely available for redistribution 